\title{
Mutant hypoxia-inducible factor $1 \alpha$ modified bone marrow mesenchymal stem cells ameliorate cerebral ischemia
}

\author{
CHUNYU YANG ${ }^{1}$, HUA LIU $^{2}$ and DANPING LIU ${ }^{3}$ \\ Departments of ${ }^{1}$ Neurosurgery, ${ }^{2}$ Ophthalmology and ${ }^{3}$ Orthopedics, Liaoning Medical University, \\ Linghe, Jinzhou, Liaoning 121001, P.R. China
}

Received April 1,2014; Accepted September 24, 2014

DOI: $10.3892 / \mathrm{ijmm} .2014 .1953$

\begin{abstract}
Hypoxia-inducible factor $1 \alpha(\mathrm{HIF} 1 \alpha)$ plays stimulatory roles in revascularization in the ischemic area of cerebral ischemia. However, the hydroxylation of proline at 564 and asparagine at 803 in the HIF1 $\alpha$ coding sequence facilitated the degradation of HIF1 $\alpha$ and inhibited the transcription activity of the HIF1 $\alpha$ promoter under normoxic conditions and confined the pro-angiogenic efficacy of HIF1 $\alpha$. In the present study, the HIF1 $\alpha$ mutant containing P564A and N803A was constructed by site-directed mutagenesis. Rat bone marrow mesenchymal stem cells (BMSCs) were infected with adenoviral particles containing HIF1 $\alpha$ mutant at multiplicity of infection of 150 . The HIFl $\alpha$ mRNA and protein levels under hypoxia and normoxic conditions were compared using reverse transcription-polymerase chain reaction and western blot analysis. To explore the therapeutic effect of mutant HIF1 $\alpha$ on the cerebral ischemia, BMSCs overexpressing mutant HIF1 $\alpha$ were transplanted in the rat middle cerebral artery occlusion model (MCAO). The motor function and cerebral infarct size were evaluated using modified neurological severity score and triphenyltetrazolium chloride (TTC) staining within four weeks after MCAO. Vascular endothelial growth factor (VEGF) protein expression was detected by western blot analysis. Microvessel density and angiogenesis were detected by immunohistochemistry to evaluate the recovery of the brain ischemia. The HIF1 $\alpha$ mutant containing P564A and N803A could be expressed under normoxic conditions. Transplantation of BMSCs stably expressing mutant HIF1 $\alpha$ significantly improved motor function, reduced cerebral infarction and increased VEGF protein expression revascularization at days 7,14 and $28(\mathrm{P}<0.05)$. Therefore, the HIF1 $\alpha$ mutant containing P564A and N803A may be a potential target for the treatment of the cerebral ischemia.
\end{abstract}

Correspondence to: Professor Hua Liu, Department of Ophthalmology, Liaoning Medical University, No. 40, Section 3, Songpo Road, Linghe, Jinzhou, Liaoning 121001, P.R. China E-mail: hualiu0403@126.com

Key words: mutant HIF1 $\alpha$, bone marrow mesenchymal stem cells, cerebral ischemia, angiogenesis

\section{Introduction}

Ischemic cerebrovascular disease is caused by cerebral vascular stenosis or occlusion leading to cerebral ischemia or thrombosis, which accounts for $70-80 \%$ of all cerebrovascular disease. How to prevent secondary brain injury following cerebral ischemia is currently of interest in clinical and scientific research. Previous studies indicate that angiogenesis is one of the key factors in the recovery of secondary brain injury. Hypoxia-inducible factor $1 \alpha(\mathrm{HIF} 1 \alpha)$ plays an important role in angiogenesis (1-4). HIF1 $\alpha$ promotes angiogenesis without causing blood vessel leakage, tissue edema and inflammatory reaction (5), and is considered a promising therapeutic target for clinical treatments.

HIF1 $\alpha$ is an important transcription factor and a functional subunit. Its protein stability and transcriptional activity are mainly regulated by the intracellular oxygen concentration $(6,7)$. HIF1 $\alpha$ facilitates cell survival under hypoxic conditions by binding to the hypoxia response element in the target gene and regulates the expression of broad spectrum of angiogenesis-related genes, including vascular endothelial growth factor (VEGF). As a result, VEGF promotes the angiogenesis and revascularization, and improves the blood supply (8-10). However, HIF1 $\alpha$ is only expressed under hypoxia conditions and degraded rapidly under normoxic conditions due to the hydroxylation of the 564-proline and 803-asparagine in the coding sequences (CDS). Hydroxylation of the 564-proline causes HIF1 $\alpha$ degradation by the oxygen-dependent degradation domain (ODDD) (11-14). The 803-asparagine in the C-terminal region (COOH-terminal transactivation domain, CAD) determines the HIF1 $\alpha$ transcriptional activity. The prevention of 803-asparagine hydroxylation with chemical inhibitors or genetic recombination can induce strong transcription activity of HIF1 $\alpha$ (15).

Thus far, there are few studies that focus on the treatment of cerebral ischemia using mutant HIF1 $\alpha$-transfected BMSCs. In the present study, the 564-proline and 803-asparagine in the CDS were simultaneously mutated into alanine and the mutant HIF1 $\alpha$ recombinant adenovirus vector was constructed. The HIFla gene recombinant vector was transfected into BMSCs (16), and subsequently the infected BMSCs were transplanted to the middle cerebral artery occlusion model (MCAO) to achieve high levels of HIF1 $\alpha$ protein expression and promote revascularization in the ischemic area. 


\section{Materials and methods}

Animals. Male Sprague Dawley (SD) rats (280-320 g, 6-8 weeks old) were provided by the Experimental Animal Center of Liaoning Medical College (Jinzhou, Liaoning, China). All the experimental procedures were carried out according to the internationally accredited guidelines with the approval of the Institutional Animal Care and Use Committee of Drug Safety Evaluation Center of Liaoning Medical College.

Reagents. The recombinant adenovirus vector containing wild-type HIF1 $\alpha$ or mutant HIF1 $\alpha$ was kindly given by Danping Liu (Liaoning Medical College).Lipofectamine 2000 and Opti-MEM were purchased from Invitrogen Life Technologies (Carlsbad, CA, USA); pMD19-T and reverse transcription-polymerase chain reaction (RT-PCR) kit were purchased from Takara Bio, Inc. (Otsu, Japan); PmeI and PacI were purchased from New England Biolabs, Inc. (Ipswich, MA, USA); Dulbecco's modified Eagle's medium (DMEM), trypsin and fetal bovine serum (FBS) were purchased from Gibco Life Technologies (Carlsbad, CA, USA); anti-HIF1 $\alpha$ (rabbit; no. 10790), anti-VEGF (rabbit; no. 507), anti-cluster of differentiation 105 (CD105) (rabbit; no. 20632), anti- $\beta$-actin (no. 130656) and the corresponding secondary antibodies [horseradish peroxidase (HRP)-conjugated; no. 2004] were purchased from Santa Cruz Biotechnology, Inc., (Santa Cruz, CA, USA); 2,3,5-triphenyltetrazolium chloride was purchased from Sigma (St. Louis, MO, USA); and the HEK293A cells were purchased from the Type Culture Collection of the Chinese Academy of Sciences (Shanghai, China).

Infection of BMSCs with recombinant adenovirus. The recombinant adenovirus vector was introduced into the HEK293A cells for packaging and infectious viral particles were obtained. Rats BMSCs were isolated and purified according to the adhesion method. BMSCs were cultured in low-glucose DMEM medium containing $10 \% \mathrm{FBS}$ and $1 \%$ penicillin/streptomycin at $37^{\circ} \mathrm{C}, 5 \% \mathrm{CO}_{2}$. The cells were infected (multiplicity of infection=150) and the infection efficiency was observed under fluorescence microscope after $72 \mathrm{~h}$ of infection.

Animal middle cerebral artery occlusion model and bone marrow mesenchymal stem cell transplantation. Forty-eight adult SD rats were randomly divided into four groups. The suture occlusion method was used, as previously described by Longa et al (17). In brief, a $0.2-\mathrm{mm}$ suture was inserted into the external carotid artery with a depth of $18.0 \pm 0.5 \mathrm{~mm}$ to occlude the entrance of the right middle cerebral artery to induce ischemia. Two hours after the occlusion, the suture was removed to create a reperfusion model. The rats were randomly divided into four groups and $5 \times 10^{6}$ BMSCs were injected via the tail vein $6 \mathrm{~h}$ after MCAO (18).

Evaluation of the cerebral infarction and functional outcome. Functional outcome was evaluated by the modified neurological severity score (mNSS) (19). The higher the mNSS score, the more severe the neurological dysfunction. Triphenyltetrazolium chloride (TTC) staining was carried out at days 1,14 and 28 after MCAO. The rats were sacrificed and the brains were collected. The coronal sections $(2 \mathrm{~mm})$ were incubated in $2 \% \mathrm{TTC}$ solution at $37^{\circ} \mathrm{C}$ for $15 \mathrm{~min}$. The relative infarct volume was calculated based on methods described by Neumann-Haefelin et al (20).

$R T-P C R$. Total RNA was extracted using using TRIzol (Invitrogen Life Technologies). The optical density (OD) 260/OD280 values were measured by UV spectrophotometer to assess the quality of the RNA. RNA was subsequently reverse-transcribed into cDNA by RT-PCR. The thermal cycler parameters were $95^{\circ} \mathrm{C}, 3 \mathrm{~min} ; 94^{\circ} \mathrm{C}, 30 \mathrm{sec}, 59^{\circ} \mathrm{C}, 30 \mathrm{sec}$, $72^{\circ} \mathrm{C}, 45 \mathrm{sec}, 36$ cycles; and $72^{\circ} \mathrm{C}, 7 \mathrm{~min}$; prior to storage at $4^{\circ} \mathrm{C}$. Following the reaction, $5 \mu 1$ of PCR product for each cell sample was used for $1.5 \%$ agarose gel electrophoresis. The OD of each band was analyzed by a gel imaging system (I-box; UVP, Upland, CA, USA). The experiment was carried out in triplicate and the relative OD values were independently calculated. The primers used for PCR amplification were as follows: 564 forward, CTGGCCGCAGCCGCTGGAGACAC; and reverse, GGATATAGGCAGCTAACATCTCC; 803, GATGTTAGCTGCCTATATCCCAATG; and reverse, TTCA CCCTGCAGTAGGTTTCTGCTGCCTTGTATAGGAGCA GCAACTTCACAATC; HIFl $\alpha$ forward, GAAACCACC TATGACCTGC; and reverse, GTCGTGCTGAATAA TACCACTC; $\beta$-actin forward, GGGACCTGACTGACTA CCTC; and reverse, TCATACTCCTGCTTGCTGAT.

Immunohistochemistry. The rats were decapitated and the right brain hemispheres were collected. The tissues were fixed, dehydrated, embedded in paraffin and sectioned at the coronal plane. The sections were then incubated with rabbit anti-mouse CD105 factor antibody (1:200) for immunohistochemical staining. Following the 3,3'-diaminobenzidine reaction, the sections were stained with hematoxylin. Microvessel density (MVD) was calculated based on the method described in the study by Weidener et al (21). A brown-stained single endothelial cell (or endothelial cell cluster) was used as a vessel count; six fields on each slice were randomly selected for the microvessel count. The number of vessels per unit $\left(\mathrm{mm}^{2}\right)$ (microvessel density) was calculated and averaged.

Western blot analysis. The total protein was extracted. Equal amounts of protein $(50 \mu \mathrm{g})$ were separated by $10 \%$ SDS-PAGE and transferred to a polyvinylidene fluoride membrane. The membranes were blocked with 5\% skimmed dry milk for $1 \mathrm{~h}$ at room temperature and incubated with primary antibody [anti-HIF1 $\alpha$ (rabbit) or anti-VEGF (rabbit), 1:1,000] at $4^{\circ} \mathrm{C}$ overnight. The membranes were washed and incubated with the HRP-conjugated secondary antibodies for $1 \mathrm{~h}$ at room temperature. Subsequent to washing, the membranes were incubated in developing solution in a dark room for $30 \mathrm{~min}$ at room temperature. The expression levels of targeted protein were detected by enhanced chemiluminescence and analyzed using Quantity One (Bio-Rad, Hercules, CA, USA). The experiment was carried out in triplicate and the relative OD values were calculated.

Statistical analysis. Data are presented as the means \pm standard deviation. One-way analysis of variance was used to analyze the difference among groups. $\mathrm{P}<0.05$ was considered to indicate a statistically significant difference. 

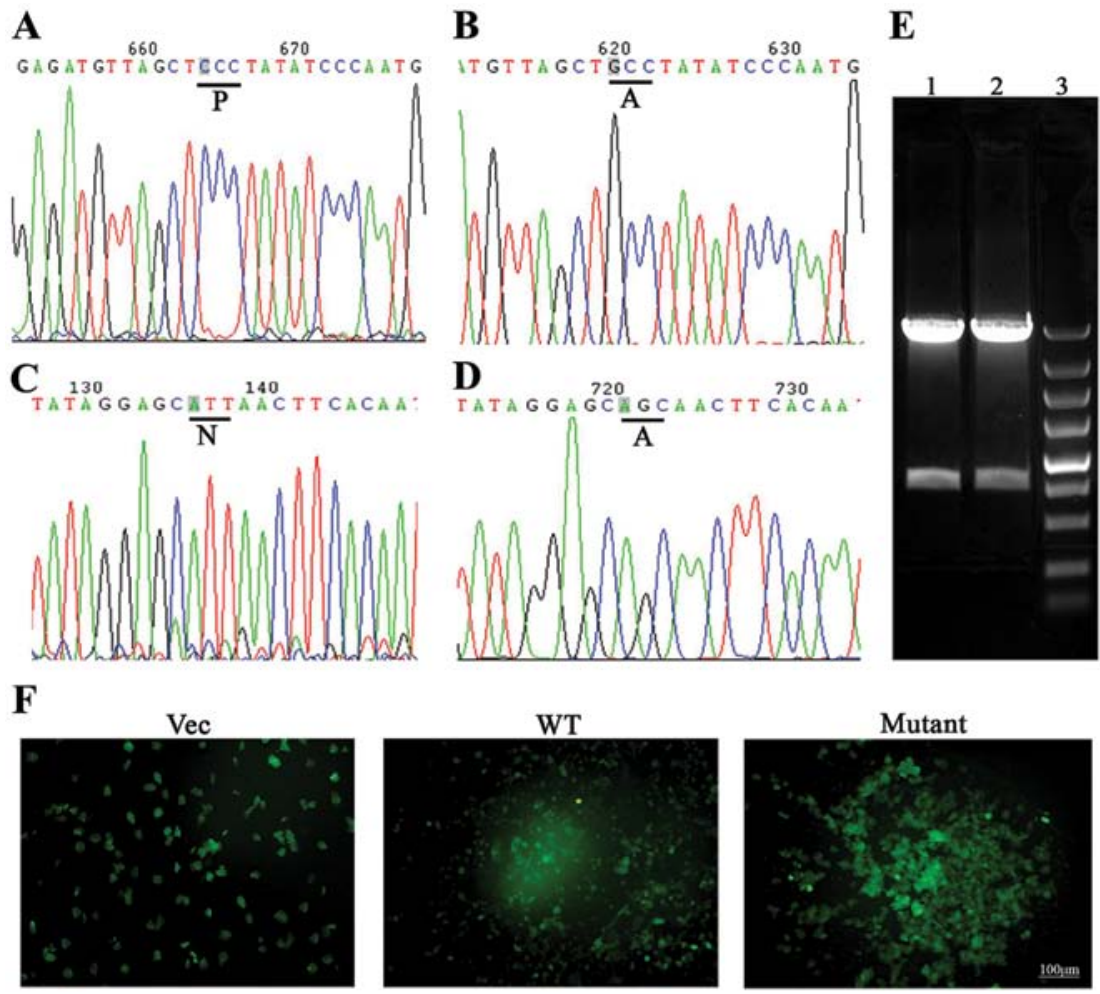

Figure 1. Identification of the HIF1 $\alpha$ mutant. (A-D) DNA sequencing analysis of the wild-type HIFl $\alpha$ and mutant HIF1 $\alpha$ gene. (A and B) C to G transversion was introduced in the CCC, which caused proline (P) at 564 to mutate into alanine (A); (C and D) the ATT was mutated into AGC and caused the asparagine (N) to mutate into alanine (A) at 803. (E) Electrophoretic map of adenovirus vector digested by PacI. Lane 1, the adenovirus vector containing wild-type HIF1 $\alpha$ gene; lane 2, the adenovirus vector containing mutated HIF1 $\alpha$ gene; lane 3, marker. (F) Virus packaging in adenovirus vector-transfected HEK293A cells (original magnification, x100). HIF1 $\alpha$, hypoxia-inducible factor $1 \alpha$.
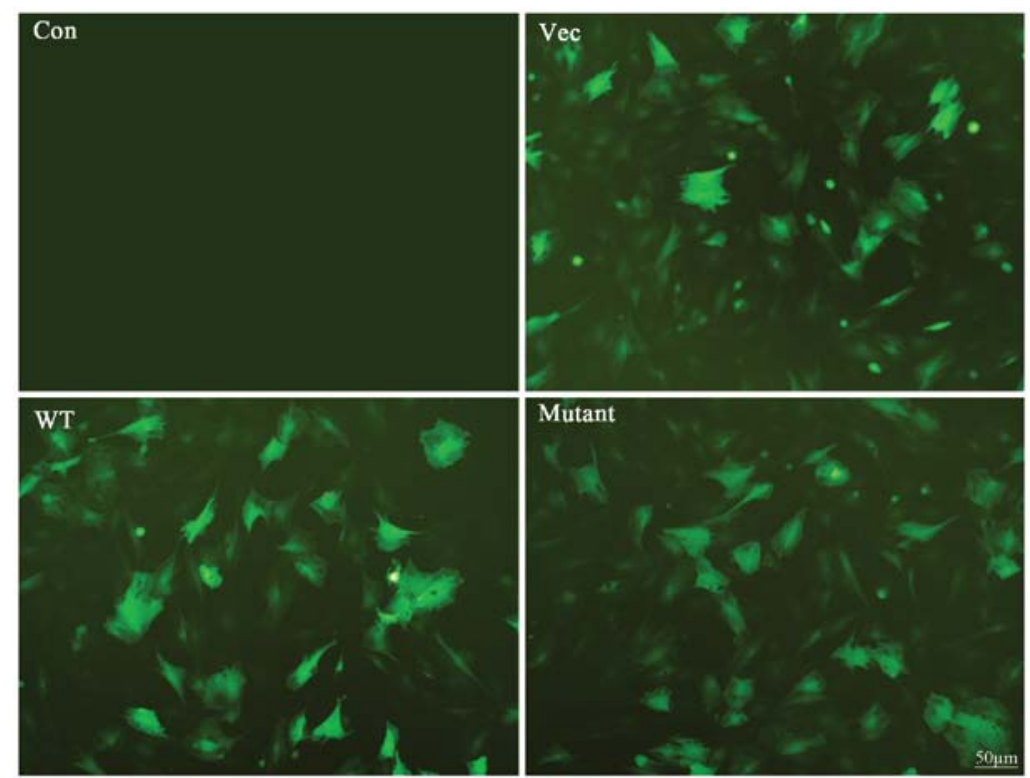

Figure 2. Fluorescence observation of the mutant HIF1 $\alpha$ expression in BMSCs. Scale bar, $50 \mu \mathrm{m}$. HIF1 $\alpha$, hypoxia-inducible factor $1 \alpha$.

\section{Results}

Infection of BMSCs with the recombinant adenovirus particles. As shown in Fig. 1A-D, DNA sequencing assay showed that the proline at 564 and the asparagine at 803 of HIF1 $\alpha$ were mutated into alanine. A 30- and a 3-kb band were observed following PacI cleavage (Fig. 1E). This mutant was transfected into HEK293A cells using Lipofectamine 2000 (Fig. 1F). The infectious recombinant adenovirus particles were harvested and the titer was $2.1 \times 10^{9} \mathrm{pfu} / \mathrm{ml}$. Subsequently, the BMSCs were infected using these virus particles containing P564A and N803A, and the infectious status was observed by an immunofluorescence microscope. HIF1 $\alpha$ - and mutant-HIF1 $\alpha$ transfected BMSCs showed strong expression of green 

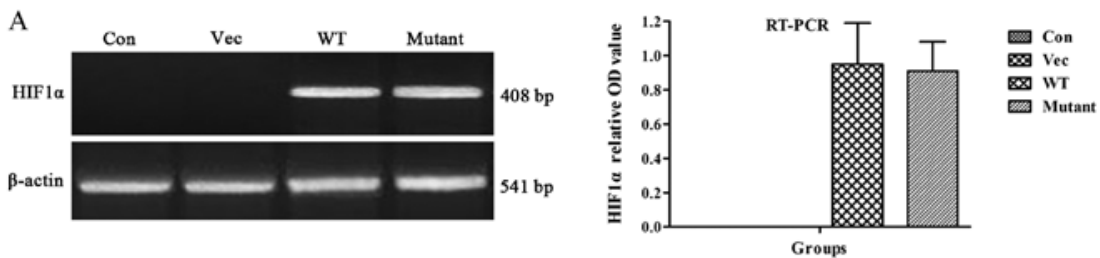

B
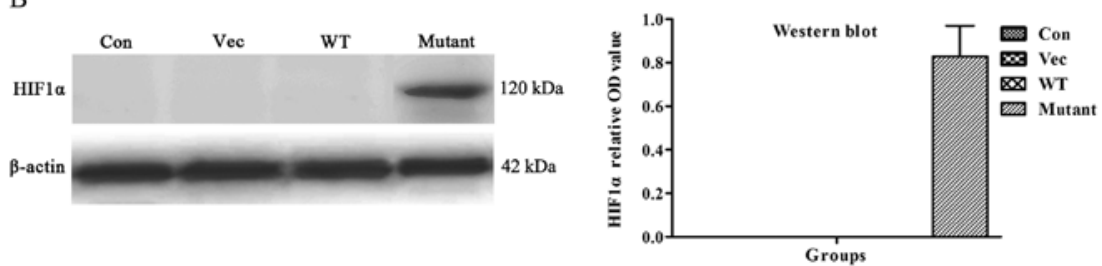

Figure 3. Expression of mutant HIF1 $\alpha$ mRNA and protein under normoxic conditions. (A) RT-PCR analysis of the mutant HIF1 $\alpha$ mRNA expression under normoxic conditions. (B) Western blot analysis of mutant HIF1 $\alpha$ protein expression under normoxic conditions. These data are represented as the mean \pm standard deviation and analyzed by one-way ANOVA. The difference is statistically significant when $\mathrm{P}<0.05$. Con, control; Vec, vector; HIF1 $\alpha$, hypoxia-inducible factor $1 \alpha$; RT-PCR, reverse transcription-polymerase chain reaction; WT, wild-type HIF1 $\alpha$; SD, standard deviation; ANOVA, analysis of variance.
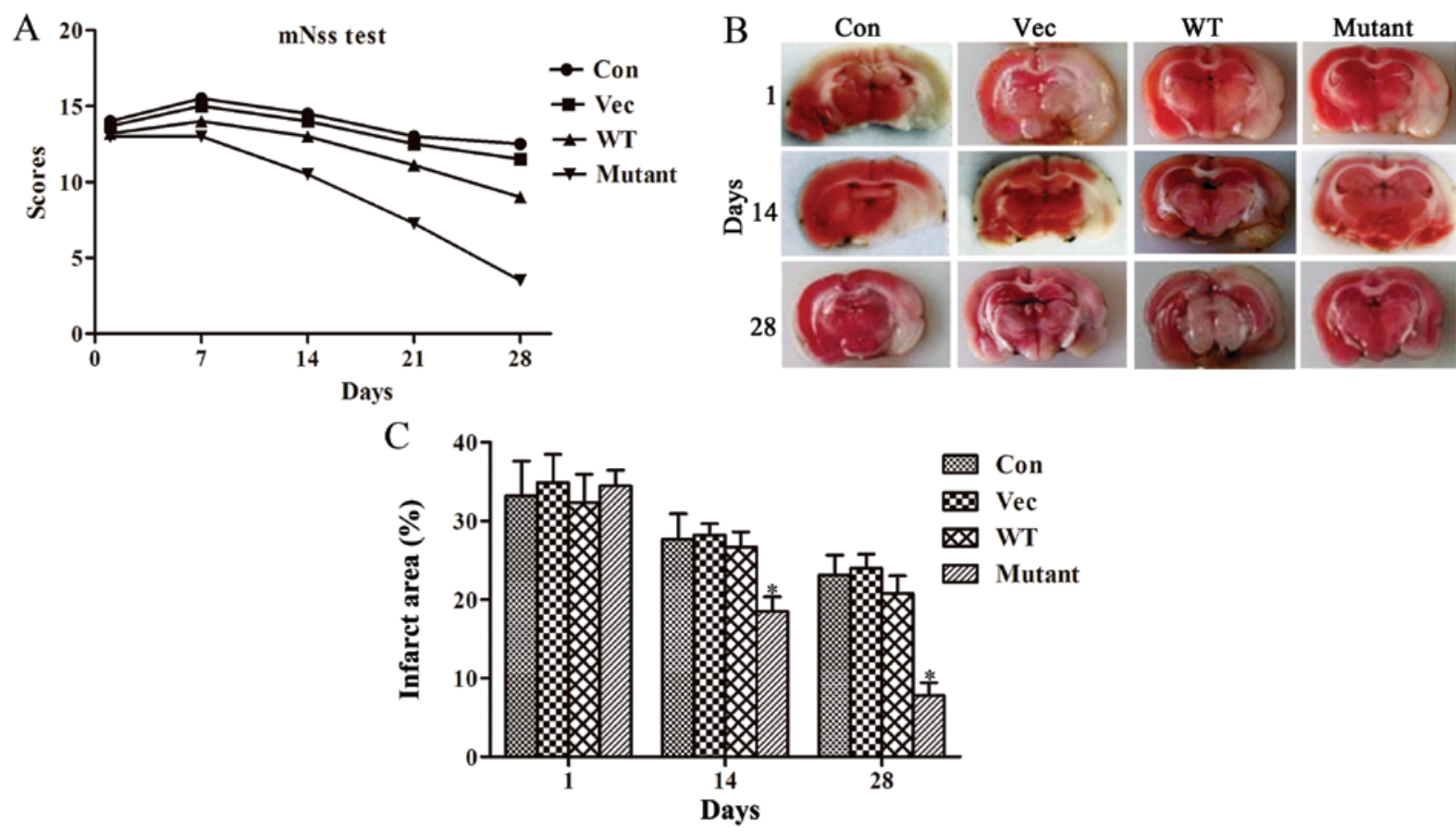

Figure 4. Mutant HIF1 $\alpha$ improved the functional outcome and decreased infarct size. (A) Neurological function evaluation at days 1, 7, 14, 21, 28 after MCAO. These experiments were repeated three times. (B) TTC staining of the infarcted area at days 1, 14 and 28 after MCAO. (C) Quantitative analysis of TTC staining $(\mathrm{P}<0.05)$. The data are represented by the mean \pm standard deviation and analyzed by one-way ANOVA. The difference is considered statistically significant when $\mathrm{P}<0.05$. HIF1 $\alpha$, hypoxia-inducible factor $1 \alpha$; Con, control; Vec, vector; WT, wild-type HIF1 $\alpha$; MCAO, middle cerebral artery occlusion model; TTC, triphenyltetrazolium chloride; ANOVA, analysis of variance.

fluorescent protein (Fig. 2) indicating that mutant HIF1 $\alpha$ can be expressed at high level under nomorxic conditions.

The expression of mutant HIF1 $\alpha$ were quantitatively examined in BMSCs at mRNA and protein levels under normoxic conditions. RT-PCR showed that the expression of mutant HIFl $\alpha$ mRNA could be detected under normoxic conditions (Fig. 3A). Western blot analysis revealed that the mutant HIF1 $\alpha$ protein was only detected under normoxic conditions. These data further indicated that the HIF1 $\alpha$ mutant could be expressed under normoxic conditions as compared to HIF1 $\alpha$, which is only expressed under hypoxia conditions (Fig. 3B).
Mutant HIFl $\alpha$ improves the functional outcome and decreases the infarct size. The mutant-HIF1 $\alpha$ therapy outcomes in rats were evaluated using the mNSS on days 1, 7, 14, 21 and 28. A lower mNSS score indicates that less neurological defects resulted from $\mathrm{MCAO}$ and represents a more improved outcome. As shown in Fig. 4A, there was a significant improvement in neurological function in the mutant-HIF1 $\alpha$ group compared to the other three groups from days 14-28 $(\mathrm{P}<0.05)$. However, no significant difference were observed in all the groups at day 1 and day 7 after MCAO $(\mathrm{P}>0.05)$. The mNSS score was significantly lower in the mutant HIF1 $\alpha$ group than the other three groups at day 14 

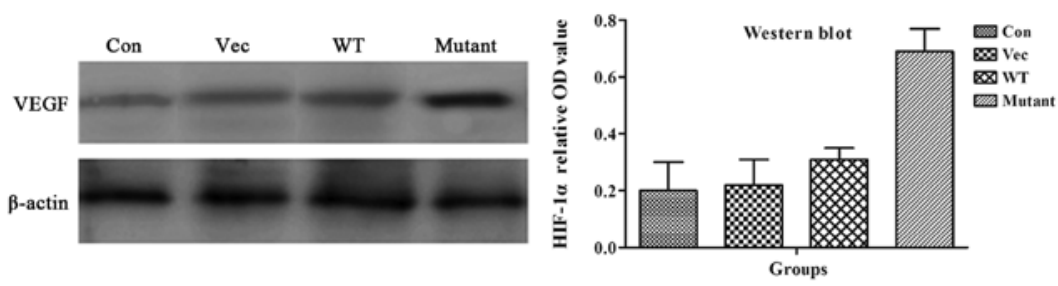

Figure 5. VEGF protein expression and revasculation in the mutant group. Western blot analysis of VEGF protein expression in the mutant HIF1 $\alpha$-treated group $(\mathrm{P}<0.05)$. All the experiments were repeated three times. The data are represented by mean \pm standard deviation and analyzed by one-way ANOVA. The difference is considered statistically significant when $\mathrm{P}<0.05$. VEGF, vascular endothelial growth factor; HIF1 $\alpha$, hypoxia-inducible factor $1 \alpha$; Con, control; Vec, vector; WT, wild-type HIF1 $\alpha$; Mutant, mutant HIF1 $\alpha$.
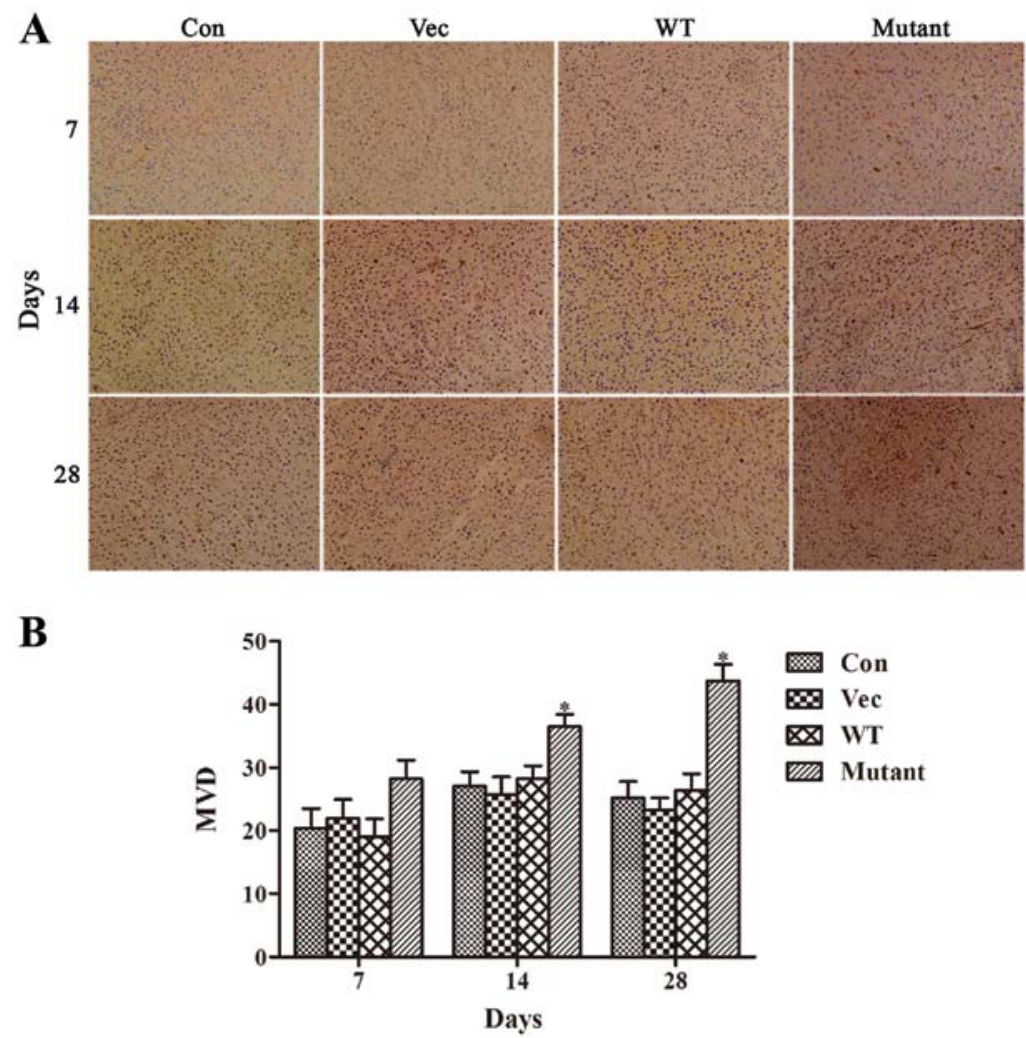

Figure 6. Mutant HIF1 $\alpha$ facilitates the formation of microvessel density. (A) Immunohistochemistry analysis of microvessel density using CD105 antibody at day 7, 14 and 28 after MCAO. (B) Quantitative analysis of the status microvessel density. All the experiments were repeated three times. The data were represented by mean \pm standard deviation and analyzed by one-way ANOVA. The difference is considered statistically significant when $\mathrm{P}<0.05$. HIF1 $\alpha$, hypoxia-inducible factor $1 \alpha$; Con, control; Vec, vector; WT, wild-type HIF1 $\alpha$; CD105, cluster of differentiation; MCAO, middle cerebral artery occlusion model; ANOVA, analysis of variance.

$(\mathrm{P}<0.05)$, whereas the scores were similar among the other three groups $(\mathrm{P}<0.05)$. Compared to the other groups, the scores of the mutant-HIF $1 \alpha$ group decreased more significantly at day 21 and day 28, indicating that the mutant HIF1 $\alpha$ could effectively mitigate motor function $(\mathrm{P}<0.05)$ (Fig. 4A). Compared to the other groups, TTC staining showed that the infarcted area decreased significantly in the mutant-HIFla groups. This indicated that mutant HIF1 $\alpha$ could attenuate cerebral ischemic injury in rats. The infarct area (white area) was similar among all the groups at day $1(\mathrm{P}<0.05)$. On day 14 , the infarct size in the mutant-HIF1 $\alpha$ group was significantly reduced, compared to the other three groups $(\mathrm{P}<0.05)$; at day 28, the reduction in the infarct size became clearer in comparison to the other groups $(\mathrm{P}<0.05)$, indicating that mutant HIF1 $\alpha$ effectively reduced ischemic injury in rats (Fig. 4B and C).
VEGF protein expression and revasculation in the mutant group. Western blot analysis showed that VEGF protein expression was significantly higher in the mutant-HIFl $\alpha$ group than the other three groups $(\mathrm{P}<0.05)$, indicating that the mutant HIF $1 \alpha$ significantly increased the expression of the downstream gene, VEGF, which was consistent with the mutant-HIF1 $\alpha$ function under normoxic conditions (Fig. 5).

The number of new blood vessels at the ischemia areas were also evaluated at the same time. For the wild-type HIF1 $\alpha$ group, a small amount of scattered microvascular could be observed at 7 days after cerebral ischemia. The revasculation peaked at day 14 after ischemia and subsequently decreased gradually. Whereas, the mutant-HIF1 $\alpha$ group showed that the density of neomicrovascular vessels was denser at day 7 , peaked at day 14 and maintained at a relatively higher level at day 28 after MCAO (Fig. 6). 


\section{Discussion}

In the present study, a recombinant adenovirus HIF1 $\alpha$ was established in which the proline at 564 and asparagine at 803 were mutated simultaneously and explored the significance in the clinical treatment of cerebral ischemia. Numerous data have demonstrated that the evidence indicates that the 564-proline and the 803-asparagine are important loci that regulate HIF1 $\alpha$ expression. The 564-proline in the ODDD of HIF $1 \alpha$ is degraded by proline hydroxylase and hydroxylation of the 803-asparagine can inhibit HIF1 $\alpha$ transcriptional activity under normoxic conditions (11-15). For these reasons, HIF1 $\alpha$ accumulates only under hypoxia conditions. In the present study, the $\mathrm{P}$ to A mutation at 564 prevented oxygen-dependent degradation of HIF1 $\alpha$ and the $\mathrm{N}$ to A mutation of the 803 -asparagine increased transcriptional activity of HIF1 $\alpha$, which all lead to a stable HIF1 $\alpha$ expression under normoxic conditions. The present data have demonstrated that the mutant HIF1 $\alpha$ could be expressed at a relatively higher level under normoxic conditions. In addition, the mutant HIF $1 \alpha$ is more stable compared to wild-type HIF1 $\alpha$ under normoxic conditions with a higher expression level without degradation.

HIFl $\alpha$ mRNA expression, but not protein expression, was found under normoxic conditions, which indicates that wild-type HIF1 $\alpha$ protein can be completely degraded under normoxic conditions. However, the mutant HIF1 $\alpha$ was expressed under normoxic conditions. These data indicated that the degradation of wild-type HIF1 $\alpha$ occurs at the post-transcriptional translation stage.

BMSCs were infected with adenovirus particles containing the mutant-HIFl $\alpha$ gene in vitro and found that transfection with the mutant HIF1 $\alpha$ effectively induced the expression of HIF1 $\alpha$ under normoxic conditions, which provided a foundation for our in vivo animal experiments. In addition, VEGF protein expression in the animals treated with BMSCs overexpressing the mutant HIF1 $\alpha$ were significantly higher than that of the animals treated with BMSCs overexpressing the wild-type HIF1 $\alpha$. VEGF is a HIF $1 \alpha$-downstream gene, indicating that mutant HIF $1 \alpha$ is not only expressed under normoxic conditions, but also effectively promotes downstream gene expression, including $V E G F$, which is consistent with the wild-type HIF1 $\alpha$ function under hypoxic conditions.

Immunohistochemistry results showed that the MVD was significantly higher in the animals treated with BMSCs overexpressing the mutant HIF1 $\alpha$ at days 7, 14 and 28 compared to the other three groups, indicating that mutant HIF1 $\alpha$ promoted angiogenesis and revascularization in the cerebral ischemia area, thereby accelerate the process of motor function recovery. Correspondingly, the infarct volume was significantly reduced in the animals treated with BMSCs overexpressing the mutant HIF1 $\alpha$ than that of the animals treated with BMSCs overexpressing the wild-type HIF1 $\alpha$, which is consistent with the behavioral test results (22-24).

A previous study has shown that mutant HIF1 $\alpha$ protein can be efficiently expressed in vitro and in vivo under normoxic conditions, which accelerates the angiogenesis and the formation of microvessel system, improve local blood supply and thus, facilitate blood perfusion to ischemic brain tissue (25). Wild-type HIF1 $\alpha$ is unstable in a complex in vivo environment and less effective in promoting in vivo angiogenesis. In the present study, the unknown consequence of gene mutation was not encountered. The results showed that mutant HIF1 $\alpha$ has generated new potential for the treatment of ischemic cerebrovascular disease.

Numerous data have demonstrated that rat bone marrow mesenchymal stem cells can pass the blood brain barrier (26-29). In the present study, mutant-HIF1 $\alpha$ infected-BMSCs was injected into into rats and the BMSCs passed through the blood-brain barrier and achieved therapeutic effects. However, due to the limited experimental conditions, the amount of HIF1 $\alpha$ that successfully transferred into brain tissue was not measured. This area requires exploring further in future studies.

\section{References}

1. Giaccia A, Siim BG and Johnson RS: HIF-1 as a target for drug development. Nat Rev Drug Discov 2: 803-811, 2003.

2. Khan TA, Sellke FW and Laham RJ: Gene therapy progress and prospects: therapeutic angiogenesis for limb and myocardial ischemia. Gene Ther 10: 285-291, 2003.

3. Semenza GL: Evaluation of HIF-1 inhibitors as anticancer agents. Drug Discov Today 12: 853-859, 2007.

4. Wan C, Gilbert SR, Wang Y, et al: Activation of the hypoxia-inducible factor- $1 \alpha$ pathway accelerates bone regeneration. Proc Natl Acad Sci USA 105: 686-691, 2008.

5. Pugh CW and Ratcliffe PJ: Regulation of angiogenesis by hypoxia: role of the HIF system. Nat Med 9: 677-684, 2003.

6. Majmundar AJ, Wong WJ and Simon MC: Hypoxia-inducible factors and the response to hypoxic stress. Mol Cell 40: 294-309, 2010.

7. Wang GL, Jiang BH, Rue EA and Semenza GL: Hypoxia-inducible factor 1 is a basic-helix-loop-helix-PAS heterodimer regulated by cellular $\mathrm{O}_{2}$ tension. Proc Natl Acad Sci USA 92: 5510-5514, 1995.

8. Fiorenzo P, Mongiardi MP, Dimitri D, et al: HIF1-positive and HIF1-negative glioblastoma cells compete in vitro but cooperate in tumor growth in vivo. Int J Oncol 36: 785-791, 2010.

9. Giatromanolaki A, Fiska A, Pitsiava D, Kartalis G, Kouourakis MI and Sivridis E: Erythropoietin receptors in endometrial carcinoma as related to HIF1 $\alpha$ and VEGF expression. In vivo 23: 699-703, 2009.

10. Rey S and Semenza GL: Hypoxia-inducible factor-1-dependent mechanisms of vascularization and vascular remodelling. Cardiovasc Res 86: 236-242, 2010.

11. Bruick RK and McKnight SL: A conserved family of prolyl-4-hydroxylases that modify HIF. Science 294: 1337-1340, 2001.

12. Dang EV, Barbi J, Yang HY, et al: Control of T(H)17/T(reg) balance by hypoxia-inducible factor 1. Cell 146: 772-784, 2011.

13. Evans CE, Humphries J, Mattock K, et al: Hypoxia and upregulation of hypoxia-inducible factor $1 \alpha$ stimulate venous thrombus recanalization. Arterioscler Thromb Vasc Biol 30: 2443-2451, 2010.

14. Kazi AA, Molitoris KH and Koos RD: Estrogen rapidly activates the PI3K/AKT pathway and hypoxia-inducible factor 1 and induces vascular endothelial growth factor A expression in luminal epithelial cells of the rat uterus. Biol Reprod 81: 378-387, 2009.

15. Lando D, Peet DJ, Whelan DA, Gorman JJ and Whitelaw ML: Asparagine hydroxylation of the HIF transactivation domain a hypoxic switch. Science 295: 858-861, 2002.

16. Ralph GS, Parham S, Lee SR, et al: Identification of potential stroke targets by lentiviral vector mediated overexpression of HIF- $1 \alpha$ and HIF- $2 \alpha$ in a primary neuronal model of hypoxia. J Cereb Blood Flow Metab 24: 245-258, 2004.

17. Longa EZ, Weinstein PR, Carlson S, Cummins R, et al: Reversible middle cerebral artery occlusion without craniectomy in rats. Stroke 20: 84-91, 1989.

18. Liu N, Zhang Y, Fan L, et al: Effects of transplantation with bone marrow-derived mesenchymal stem cells modified by Survivin on experimental stroke in rats. J Transl Med 9: 105, 2011.

19. Boltze J, Kowalski I, Geiger K, et al: Experimental treatment of stroke in spontaneously hypertensive rats by $\mathrm{CD} 34^{+}$and $\mathrm{CD} 34$ cord blood cells. Ger Med Sci 3: Doc09, 2005. 
20. Neumann-Haefelin T, Kastrup A, de Crespigny A, Yenari MA Ringer T, Sun GH and Moseley ME: Serial MRI after transient focal cerebral ischemia in rats: dynamics of tissue injury, blood-brain barrier damage, and edema formation. Stroke 31: 1965-1972, 2000.

21. Weidner N, Semple JP, Welch WR and Folkman J: Tumor angiogenesis and metastasis-correlation in invasive breast carcinoma. N Engl J Med 324: 1-8, 1991.

22. Mustafa AK, Ahmad AS, Zeynalov E, et al: Serine racemase deletion protects against cerebral ischemia and excitotoxicity. J Neurosci 30: 1413-1416, 2010.

23. Ruiz-CrespoS,Trejo-Gabriel-Galán JMandComa-del-CorralMJ: Localizing coordinates of cerebral ischemic tissue without the need of staining in a rat model of focal cerebral infarct. Metab Brain Dis 28: 21-24, 2013.

24. Yu J, Zhu H, Ko D and Kindy MS: Motoneuronotrophic factor analog GM6 reduces infarct volume and behavioral deficits following transient ischemia in the mouse. Brain Res 1238 $143-153,2008$
25. Elson DA, Thurston G, Huang LE, Ginzinger DG, McDonald DM, Johnson RS and Arbeit JM: Induction of hypervascularity without leakage or inflammation in transgenic mice overexpressing hypoxia-inducible factor- $1 \alpha$. Genes Dev 15: 2520-2532, 2001

26. Menge T, Zhao Y, Zhao J, et al: Mesenchymal stem cells regulate blood-brain barrier integrity through TIMP3 release after traumatic brain injury. Sci Transl Med 4: 161ra150, 2012.

27. Pati S, Khakoo AY,Zhao J, et al: Human mesenchymal stem cells inhibit vascular permeability by modulating vascular endothelial cadherin/ $\beta$-catenin signaling. Stem Cells Dev 20: 89-101, 2011.

28. Warach S and Latour LL: Evidence of reperfusion injury, exacerbated by thrombolytic therapy, in human focal brain ischemia using a novel imaging marker of early blood-brain barrier disruption. Stroke 35: 2659-2661, 2004.

29. Zacharek A, Chen J, Cui X, et al: Angiopoietin1/Tie2 and VEGF/Flk1 induced by MSC treatment amplifies angiogenesis and vascular stabilization after stroke. J Cereb Blood Flow Metab 27: 1684-1691, 2007. 